\title{
Concentration of Kaluza-Klein dark matter in the Galactic center: constraints from gamma-ray signals
}

\author{
Satoshi Tsuchida* \\ Department of Physics, Osaka City University, 3-3-138 Sugimoto, Sumiyoshi-ku, Osaka City, \\ Osaka, 558-8585, Japan \\ E-mail: tutti125efc.ritsumei.ac.jp

\section{Masaki Mori ${ }^{\dagger}$} \\ Department of Physical Sciences, Ritsumeikan University, Kusatsu 525-8577, Shiga, Japan \\ E-mail: morimefc.ritsumei.ac.jp
}

\begin{abstract}
The Lightest Kaluza-Klein particle (LKP), which appears in the Universal Extra Dimension theory (UED), emits gamma-ray directly or secondarily, when it annihilates. The gamma-ray signal from annihilation of LKP will create prominent structure around the LKP mass, and we expect the structure can be observed by using ongoing and near-future space-based detectors with a few percent energy resolution. On the other hand, ground-based detectors, such as HESS, have 15$20 \%$ energy resolution, so we could not see a peak structure in the gamma-ray spectrum from LKP annihilation. However, by using HESS observational data, even if the peak structure does not seem to appear in the gamma-ray spectrum, we can set constraints on the boost factor, which determines the density distribution and the annihilation cross section of dark matter in the Galaxy. In addition, we discuss how we can set constraints on the boost factor, when the high accuracy gamma-ray observational data is obtained by space-based detector.
\end{abstract}

35th International Cosmic Ray Conference - ICRC2017

10-20 July, 2017

Bexco, Busan, Korea

\footnotetext{
* Corresponding Author

† Speaker.
} 


\section{Introduction}

A large fraction of the matter in the Universe is occupied by dark matter. At present, one feasible candidate of cold dark matter (CDM) is the weakly interacting massive particles (WIMPs). The density of CDM is given by $\Omega_{\mathrm{CDM}} h^{2}=0.1187 \pm 0.0017$ [四], where $\Omega_{\mathrm{CDM}}$ is the CDM density parameter of the Universe expressed as a fraction of the critical density for a flat universe, and $h$ is the Hubble constant in units of $100 \mathrm{~km} \mathrm{~s}^{-1} \mathrm{Mpc}^{-1}$.

New particles predicted by the theory of universal extra dimensions (UED) are called KaluzaKlein (KK) particles. Here, we consider the theory of UED containing only one extra dimension, which is compactified with radius $R$. We assume that the lightest KK particle (LKP) is a feasible candidate for dark matter, and we denote it $B^{(1)}$, which is the first KK mode of the hypercharge gauge boson. Because dark matter should be electrically neutral and stable, the LKP either does not interact with the standard model particles or only weakly interacts with them. The LKP mass $m_{B^{(1)}}$ may be in the range $0.5 \mathrm{TeV} \lesssim m_{B^{(1)}} \lesssim 1 \mathrm{TeV}$ using the above value for CDM density [2].

There are some LKP annihilation modes which contain gamma-rays as final products. These include gamma-ray "lines" from two-body decays, and "continuum" emission from decay or fragmentation of secondaries. The cross section for $B^{(1)}$ pair annihilation has been calculated [3], and we assume the mass splitting is $5 \%$ at the first KK level. In addition, branching ratios into these modes can be calculated for $B^{(1)}$ pair annihilation [, 团, []], which are given as follows: $20 \%$ for charged leptons, $11 \%$ for up-type quarks, $0.7 \%$ for down-type quarks, $1 \%$ for charged gauge bosons, and $0.5 \%$ for neutral gauge bosons [ $[\square, 6]$. This paper considers three patterns for the continuum: $B^{(1)}$ pairs annihilate into (i) quark pairs, (ii) charged lepton pairs which cascade or produce gamma-rays, and (iii) two leptons and one photon $\left(l^{+} l^{-} \gamma\right)$. When $B^{(1)}$ pairs directly annihilate into photon pairs, they appear as a line at the $m_{B^{(1)}}$ in the gamma-ray spectrum, which is the most prominent signal of KK dark matter. This study focuses on the detectability of this "line" structure by near-future detector taking account of their finite energy resolution.

The gamma-ray flux from annihilation of dark matter particles in the Galactic halo can be written as [四]

$$
\Phi_{\gamma}\left(E_{\gamma}, \psi\right)=\frac{\langle\sigma v\rangle}{8 \pi M^{2}} \sum_{i} B_{i} \frac{d N_{\gamma}^{i}}{d E_{\gamma}} \int_{\text {line-of-sight }} \rho^{2}(l) d l(\psi),
$$

where $M$ is the dark matter mass, $B_{i}$ is the branching ratio into the tree-level annihilation final state $i$, the function $\rho(l)$ is the dark matter density along the line-of-sight $l(\psi)$, where $\psi$ is the angle with respect to the Galactic center, $d N_{\gamma}^{i} / d E_{\gamma}$ is the gamma-ray spectrum generated per annihilation, and $\langle\sigma v\rangle$ is the total averaged thermal cross section multiplied by the relative velocity of particles.

Now, we define a "boost factor", $B_{f}$, which describes the signal enhancement from dark matter annihilation in the Galactic halo [8]. $N$-body simulation study given by Navarro-Frenk-White (NFW) [9], for example, indicates a large $B_{f}$. The boost factor $B_{f}$ is defined as following expression

$$
B_{f}=B_{\rho} \times B_{\sigma v}=\left(\frac{\left\langle\rho^{2}(l)\right\rangle_{\Delta V}}{\left\langle\rho_{0}^{2}(l)\right\rangle_{\Delta V}}\right)\left(\frac{\langle\sigma v\rangle}{3 \times 10^{-26} \mathrm{~cm}^{3} \mathrm{~s}^{-1}}\right)_{\Delta V},
$$

where $3 \times 10^{-26} \mathrm{~cm}^{3} \mathrm{~s}^{-1}$ is the typical cross section multiplied by velocity expected for thermal production of CDM [प]d], the volume $\Delta V$ is a diffusion scale, and $\rho_{0}(l)$ is a typical CDM density profile along a line-of-sight, $l$. 
Near-future missions equipped with high-energy-resolution gamma-ray detectors may resolve this issue as we discuss in the following. They could be space-based calorimetric detectors like the Calorimetric Electron Telescope (CALET) [Ш], प2], Wukong (DAMPE) [ए3] or GAMMA400 [ㄴ4]. Former two detectors have been in orbit since 2015 August and 2015 December, respectively, and the last one is planned to be launched in the middle of 2020s. These have a percent-level energy resolution and a detection area of one to several thousand square centimeters. The smallness of the area, compared with atmospheric Cherenkov telescopes, can be compensated by a longer exposure time in orbit.

In this paper, we analyze the gamma-ray spectral features from $B^{(1)}$ pair annihilation taking account of the finite energy resolution of gamma-ray detector and purposefully discuss the observability of the "line" at the $m_{B^{(1)}}$. We then give possible constraints on the boost factor by near-future detector.

\section{The effect of energy resolution}

Here, we discuss the effect of energy resolution of detectors. If the measured energy dispersion for mono-energetic gamma-rays behaves as a Gaussian distribution and the energy resolution of the detector is finite, the measured gamma-ray spectrum is blurred.

In addition, we analyze how the "line" from the $B^{(1)}$ pair annihilation into photon pairs looks above the "continuum". In Fig. 四, the solid line shows the continuum component only with an energy resolution of $1 \%$, and the patterned lines show "line" plus "continuum" spectra for different energy resolutions: the dotted line, dashed line and dot-dashed line show the spectra when the energy resolution is $0.5 \%, 1 \%$ and $2 \%$ with the Gaussian distribution respectively, assuming the boost factor $B_{f}=100$. For $2 \%$ energy resolution, the peak structure is difficult to see.

To investigate the tendency of the line component quantitatively, we consider the line to continuum ratio, which is referred to as "Line Fraction $(L F)$ ". $L F$ is defined as

$$
L F=\frac{\sum_{i} F_{i}^{l}}{\sum_{i} F_{i}^{c}},
$$

where $F_{i}^{c}, F_{i}^{l}$ are the fluxes of the continuum component and the line component of the $i$-th energy bin, respectively, and the energy bin width is set to $0.5 \mathrm{GeV}$. The summation runs from the lower to the upper energy limit of the observed line. This range is taken as $-3 \sigma_{E}$ to $+3 \sigma_{E}$ for each $m_{B^{(1)}}$, since the flux above $m_{B^{(1)}}$ drops rapidly. The result is shown in Fig. $\square$ as a function of $m_{B^{(1)}}$. In this figure, we can see that the value of $L F$ increases as $m_{B^{(1)}}$ becomes heavier, which implies characteristic peak structure is clearer for heavier $m_{B^{(1)}}$.

We can transform the spectra into counts to be observed by gamma-ray detectors. This is accomplished through multiplying by a factor of $3 \times 10^{6} \mathrm{~m}^{2} \mathrm{~s}$ for an assumed observation time of $1 \mathrm{yr}=3 \times 10^{7} \mathrm{~s}$ and an assumed effective area of $0.1 \mathrm{~m}^{2}$. These values arise from the typical aforementioned CALET sensitivity [12]. Then, we vary the mass from $500 \mathrm{GeV}$ to $1000 \mathrm{GeV}$ in $100 \mathrm{GeV}$ intervals, and calculate the count spectrum for each mass. Here, we do not consider for the systematic error, because the shape of spectral features will not be changed. The results are shown in Fig. [1], which shows that the characteristic peak structure is visually clearer when $m_{B^{(1)}}$ is heavier. That is, the line component becomes relatively larger since the continuum component decreases for heavier $m_{B^{(1)}}$. 


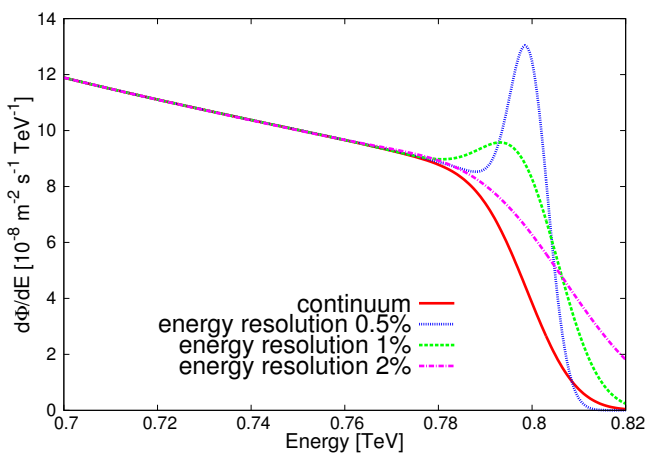

Figure 1: (Color Online) Gamma-ray spectra of continuum plus line diffused by the energy resolution assuming $m_{B^{(1)}}=800 \mathrm{GeV}$. The solid line shows the continuum component only, assuming the energy resolution of $1 \%$, while the dotted, dashed and dot-dashed lines show the continuum plus line components assuming energy resolution values of $0.5 \%, 1 \%$ and $2 \%$ respectively. The assumed boost factor is 100 .

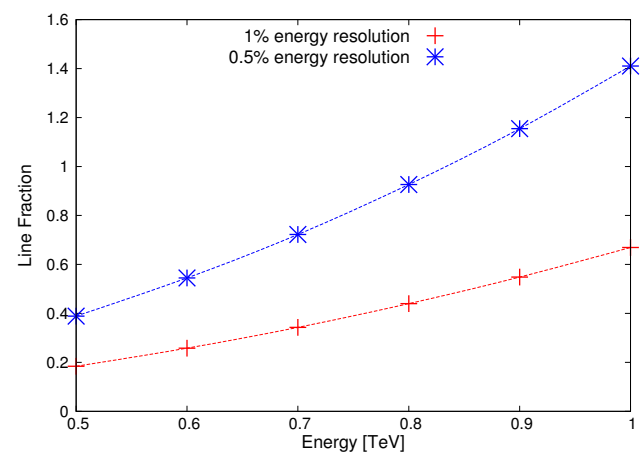

Figure 2: (Color Online) The line fraction as a functions of $m_{B^{(1)}}$, assuming an energy resolution of $0.5 \%$ and $1 \%$. The dashed curve is drawn to guide to the eyes.

\section{Discussion}

We now discuss the observability of the LKP signal in near-future detectors, taking account of present gamma-ray observations. That is, we give estimates for the accessible range of the boost factor based on upper limits on extra component in energy spectra. Here, we consider the gammaray spectrum of HESS J1745-290 located near the center of the Galaxy. This gamma-ray source can be identified as the Galactic center, Sgr A* [प5]]. Its energy spectrum above $200 \mathrm{GeV}$ is given by [1]6]

$$
\begin{aligned}
\frac{d \Phi}{d E}= & \left(2.55 \pm 0.04_{\text {stat. }} \pm 0.37_{\text {syst. }}\right)\left(\frac{E}{\mathrm{TeV}}\right)^{-2.14 \pm 0.02_{\text {stat. }} \pm 0.10_{\text {syst. }}} \\
& \times \exp \left[-\frac{E}{\left(10.7 \pm 2.0_{\text {stat. }} \pm 2.1_{\text {syst. }}\right) \mathrm{TeV}}\right] \times 10^{-8} \mathrm{TeV}^{-1} \mathrm{~m}^{-2} \mathrm{~s}^{-1}
\end{aligned}
$$

Note that with the energy resolution of HESS (15-20\%), which is a system of atmospheric Cherenkov telescopes on the ground, the LKP "line" signal is broadened and hard to detect, but the "continuum" signal could produce some structure in the energy spectrum as below.

Now, we investigate the upper limit on the boost factor, $B_{f}$, with the HESS J1745-290 spectrum. To do this, we assume the spectrum is composed of a background spectrum, represented by a power-law with an exponential cutoff, and a LKP signal. Then we employ the least-squares method to set limits on $B_{f}$. Thus the model spectrum is given by

$$
\frac{d \Phi_{\gamma}}{d E_{\gamma}}=\frac{d \Phi_{\gamma}^{\mathrm{Bkgd}}}{d E_{\gamma}}+B_{f} \frac{d \Phi_{\gamma}^{\mathrm{LKP}}}{d E_{\gamma}}
$$




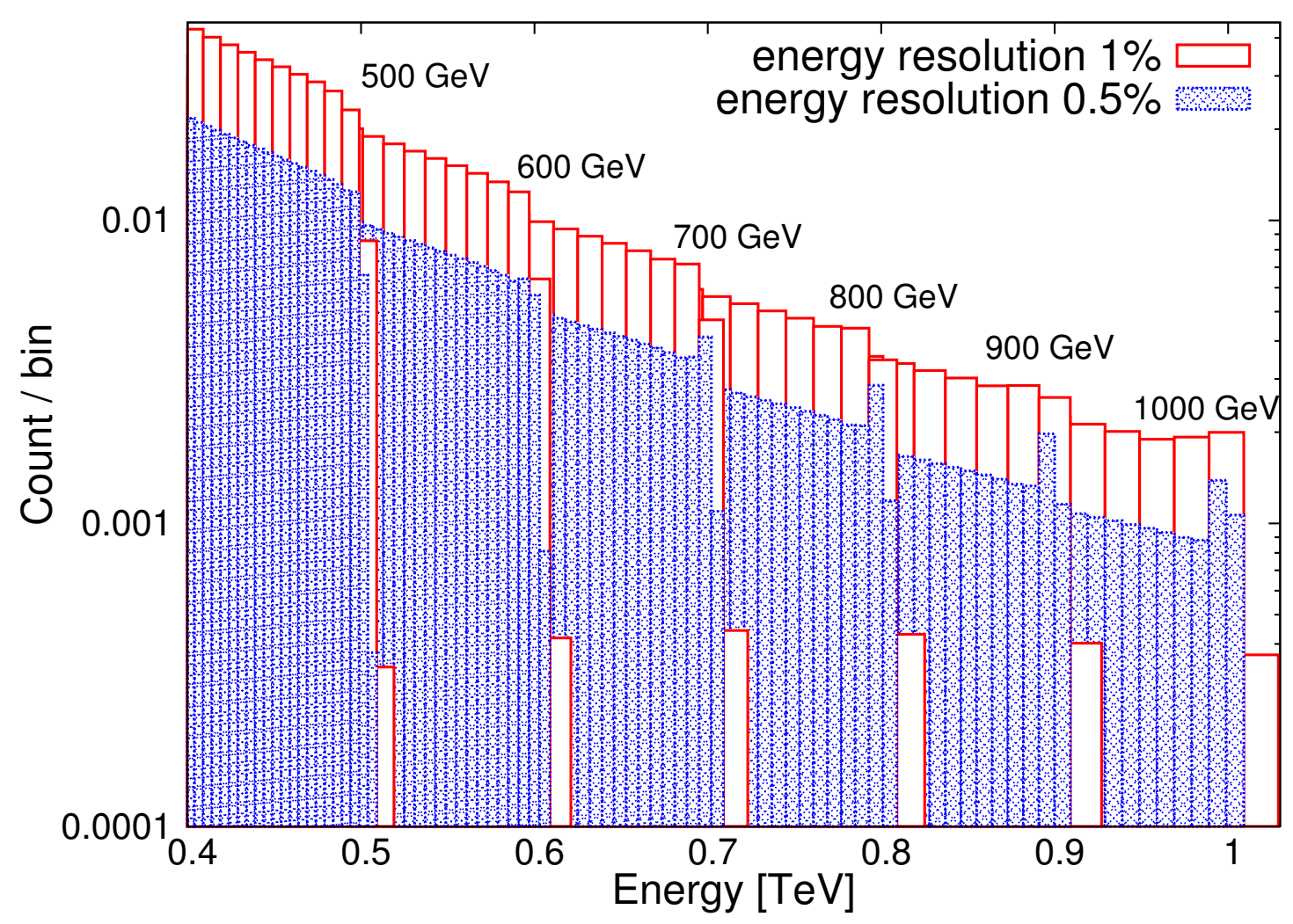

Figure 3: (Color Online) Expected count spectra, assuming energy resolutions of $0.5 \%$ and $1 \%$, and $3 \times$ $10^{6} \mathrm{~m}^{2} \mathrm{~s}$ exposure. The data spaces are twice as much as $0.5 \%$ and $1 \%$ of the $m_{B^{(1)}}$. The assumed boost factor is 100 .

with a background gamma-ray spectrum, $d \Phi_{\gamma}^{\mathrm{Bkgd}} / d E_{\gamma}$ :

$$
\frac{d \Phi_{\gamma}^{\mathrm{Bkgd}}}{d E_{\gamma}}=C_{B}\left(\frac{E}{\mathrm{TeV}}\right)^{\Gamma_{B}} \exp \left[-\frac{E}{10.7 \mathrm{TeV}}\right] \times 10^{-8} \mathrm{~m}^{-2} \mathrm{~s}^{-1} \mathrm{TeV}^{-1},
$$

where $C_{B}$ and $\Gamma_{B}$ are a coefficient and a power-law index of the background spectrum. Here we assume that the energy resolution of detector is $20 \%$, which corresponds to that of HESS, so the "LKP Flux", $d \Phi_{\gamma}^{\mathrm{LKP}} / d E_{\gamma}$, has the line plus continuum components blurred by $20 \%$ energy resolution. The goodness of the model fit to the HESS data can be tested by the sum

$$
\chi^{2}=\sum_{i} \frac{(\text { data }- \text { model })_{i}^{2}}{\sigma_{i}^{2}}
$$

where "data" is the HESS data points, $\sigma_{i}$ is its error, and "model" is given by Eq. (B.2). The index $i$ runs the data points in the energy range between about $200 \mathrm{GeV}$ to $20 \mathrm{TeV}$. A number of degrees of freedom is 22 (= a number of data points (25) minus unknown parameters $\left(3=C_{B}, \Gamma_{B}\right.$ and $\left.B_{f}\right)$ ). Thus, $\chi^{2}<40.3$ is required for the fit to be consistent with the HESS data at $99 \%$ confidence level. We calculate $\chi^{2}$ values for various parameter sets, where we vary the coefficient $C_{B}$ from 2.05 to 2.55 in 0.01 step, the index $\Gamma_{B}$ from -2.25 to -1.75 in 0.01 step, and the boost factor $B_{f}$ from 


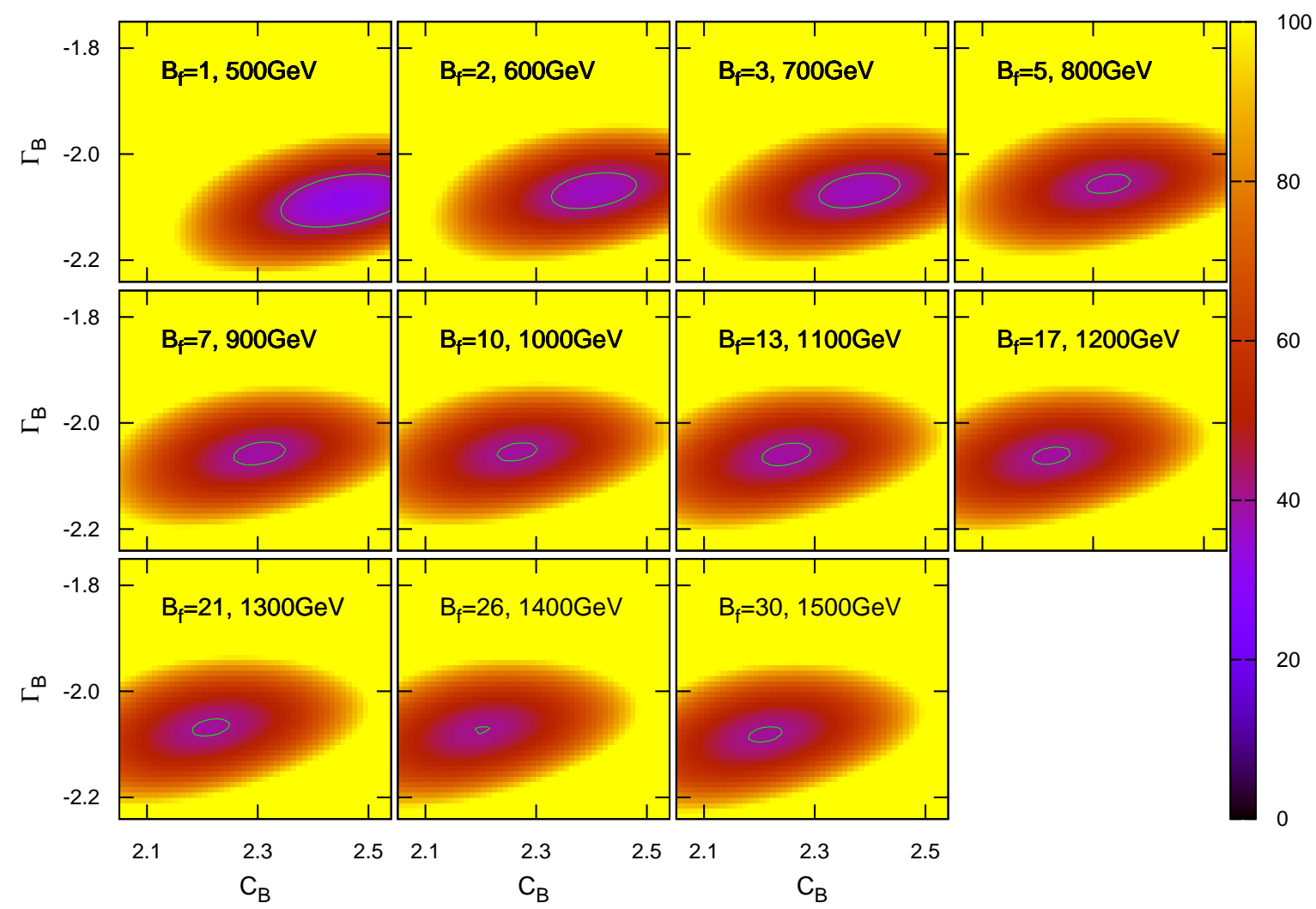

Figure 4: (Color Online) The $\chi^{2}$ values for the model fit as a function of $C_{B}$ and $\Gamma_{B}$ for each LKP mass. The region enclosed by contour indicates the region, which satisfies the condition $\chi^{2}<40.3$, and $B_{f}$ means the maximally allowed boost factor to fit HESS observational data at $99 \%$ confidence level.

0 to 100 in 1 step. The results for this calculation are shown in Fig. 团, where the $\chi^{2}$ values are plotted as a function of $C_{B}$ and $\Gamma_{B}$ for each $m_{B^{(1)}}$. The regions enclosed by contours indicate the regions which satisfy the condition $\chi^{2}<40.3$, and the values of $B_{f}$ shown in the figure mean the maximally allowed boost factors to fit HESS observational data at $99 \%$ confidence level.

On the other hand, we can think of the case that gamma-rays are detected by space-based calorimetric detectors like CALET or Wukong (DAMPE). With their fine energy resolution, we expect that the peak structure can be detected around $m_{B^{(1)}}$. When a large number of gammaray events with $0.5 \%$ energy resolution are accumulated, we may see the LKP plus background spectra as shown in Fig. [1. In this figure, the thin solid, dashed and dot-dashed lines are the model fits compatible with the HESS data at $99 \%$ confidence level with $\left(C_{B}, \Gamma_{B}, B_{f}\right)=(2.35,-2.09,1)$, $(2.29,-2.06,5),(2.23,-2.06,10)$ for $m_{B^{(1)}}=500,800,1000 \mathrm{GeV}$, respectively. The thick solid, dashed and dot-dashed lines show the spectra to be observed with $0.5 \%$ energy resolution for the same parameter sets for $C_{B}, \Gamma_{B}$ and $B_{f}$ as thin lines.

The relevant value for the boost factor, $B_{f}$, to explain the total electron plus positron spectrum observed by AMS-02 is given in range from 0 to 240 by assuming specific dark matter halo density model, halo propagation model and $m_{B^{(1)}}=1000 \mathrm{GeV}$ [ए]]. This constraint can be comparable with our result for analysis of the gamma-ray spectrum. 


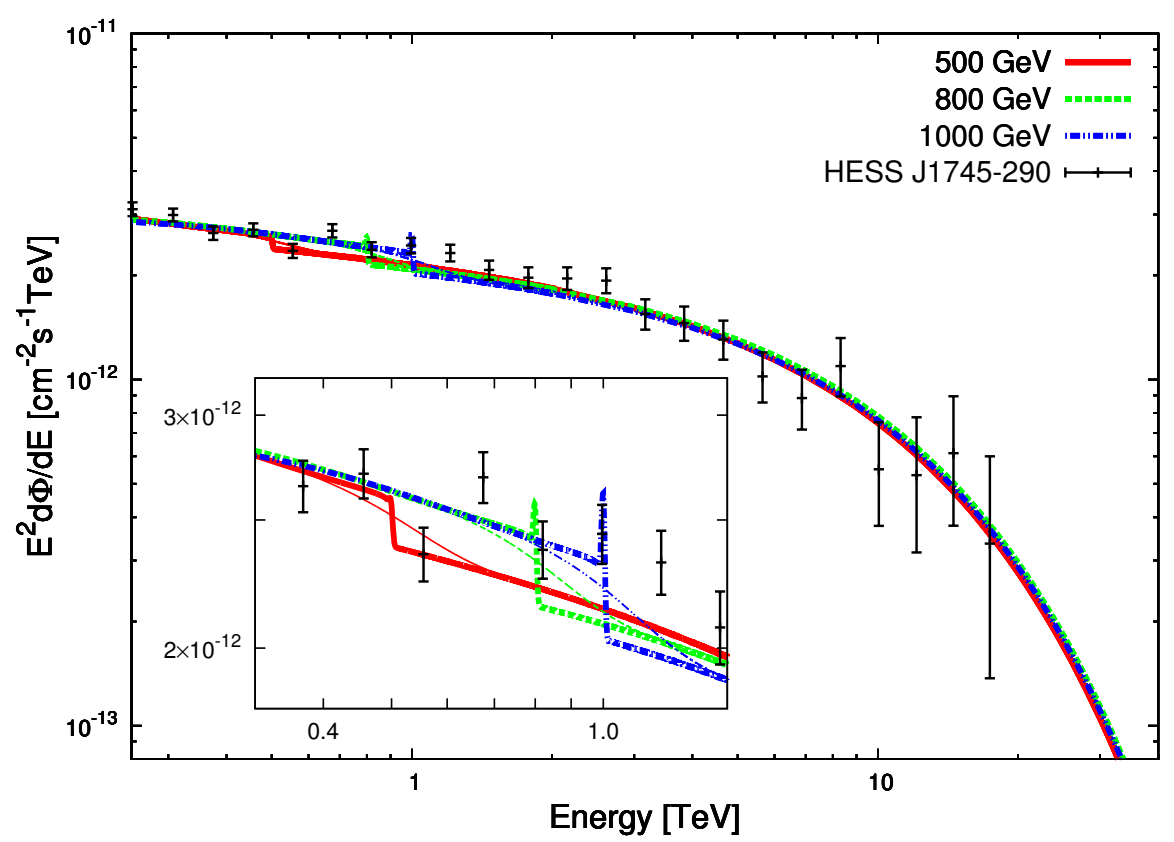

Figure 5: (Color Online) Comparison the LKP plus background spectra with HESS data (points). The thin solid, dashed and dot-dashed lines are the spectra fits to the HESS data at $99 \%$ confidence level with $\left(C_{B}, \Gamma_{B}, B_{f}\right)=(2.35,-2.09,1),(2.29,-2.06,5),(2.23,-2.06,10)$ for $m_{B^{(1)}}=500,800,1000 \mathrm{GeV}$, respectively. The thick solid, dashed and dot-dashed lines show spectra assuming $0.5 \%$ energy resolution, and the same parameter sets as thin lines.

\section{Conclusion}

In this paper, we discussed the observability of characteristic spectral feature appearing in secondarily produced gamma rays from annihilation of LKP dark matter near the Galactic center.

Energy resolution plays a key role in detecting the line structure of the gamma-ray spectral features expected from annihilation of LKP dark matter as predicted by UED theories. This paper investigated the effects of energy resolution of gamma-ray detector and calculated the expected count spectrum. The predicted gamma-ray spectrum is the sum of the continuum and the line corresponding to the LKP mass, $m_{B^{(1)}}$, but this characteristic structure is diluted when we take account of finite energy resolution of detectors. The characteristic peak indicating $m_{B^{(1)}}$ would be diffused if the energy resolution is $2 \%$ or worse as shown in Fig. $\mathrm{W}$. In addition, if $m_{B^{(1)}}$ is heavy, the observed gamma-ray spectrum will show the characteristic peak clearly because the continuum component decreases relative to the line component, as is shown in Fig. [ and Fig. [1.

The obtained upper limits on $B_{f}$ assuming the coefficient $C_{B}$ and index $\Gamma_{B}$ for the powerlaw background spectrum based on the HESS observation are given in Fig. 团. In addition, if we can detect the LKP signal with $0.5 \%$ energy resolution detector, we would see the characteristic structure around the $m_{B^{(1)}}$, as is shown in Fig. [1, when large number of photons are detected.

If the characteristic structure in gamma-ray flux is observed by new and future missions, like CALET [18], Wukong [13] and GAMMA-400 [14]], we may conclude dark matter is made of LKP. It would be a conclusive evidence for the existence of extra dimensions. 


\section{Acknowledgments}

We would like to thank Mr. Akihiko Kawamura and Dr. Fumihiro Matsui for useful discussions and helpful comments.

\section{References}

[1] P. A. R. Ade et al., Astron. Astrophys. 571, A16 (2014).

[2] L. Bergström, T. Bringmann, M. Eriksson, and M. Gustafsson, Phys. Rev. Lett. 94, 131301 (2005).

[3] L. Bergström, T. Bringmann, M. Eriksson, and M. Gustafsson, J. Cosmol. Astropart. Phys. 04, 004 (2005).

[4] H. C. Cheng, K. T. Matchev, and M. Schmaltz, Phys. Rev. D 66, 036005 (2002).

[5] G. Servant and T. M. P. Tait, Nucl. Phys. B 650, 391 (2003).

[6] D. Hooper and G. D. Kribs, Phys. Rev. D 67 (2003) 055003.

[7] A. Cesarini, F. Fucito, A. Lionetto, A. Morselli, and P. Ullio, Astropart. Phys. 21, 267 (2004).

[8] F. Prada, A. Klypin, J. Flix, M. Martinez, and E. Simonneau, Phys. Rev. Lett. 93, 241301 (2004).

[9] J. F. Navarro, C.S. Frenk, and S. D. White, Astrophys. J. 462, 563 (1996).

[10] L. Bergström, New J. Phys. 11, 105006 (2009).

[11] S. Torii, Nucl. Instrum. Methods. A 630, 55 (2011).

[12] M. Mori et al., Proc. 33rd ICRC (Rio de Janeiro), Paper 0248 (2013).

[13] V. Galio et al., Proc. 34th ICRC (The Hague), PoS (ICRC2015) 1199.

[14] N. P. Topchiev et al., Proc. 34th ICRC (The Hague), PoS (ICRC2015) 1026.

[15] M. Chernyakova, D. Malyshev, F. A. Aharonian, R. M. Crocker, and D. I. Jones, Astrophys. J. 726, 60 (2011).

[16] A. Abramowski et al. (HESS Collaboration), Nature 531, 476 (2016).

[17] S. Tsuchida and M. Mori, Int. J. Mod. Phys D, in press (2017), doi:10.1142/S021827181750095X.

[18] S. Torii for the CALET collaboration, Proc. 34th ICRC (The Hague), PoS (ICRC2015) 581. 